\title{
Acerca de la recepción del Derecho Romano en la China actual
}

\author{
About reception of Roman Law in the current China
}

\author{
José María BLANCH NOUGUÉS \\ Universidad Autónoma de Madrid
}

Resumen: El artículo aborda la importante recepción del Derecho Romano en la República Popular China a lo largo de las últimas décadas, el cual, reelaborado por la pandectística alemana, es objeto hoy en día de estudio en los planes docentes de numerosas Facultades de Derecho de aquel país y ha aportado a la doctrina china actual un universo de términos, conceptos y categorías jurídicas que se han recogido en la nueva legislación, sobre todo la relativa al Derecho civil.

\footnotetext{
Abstract: The article addresses the important reception of Roman Law in the People's Republic of China over the last decades, which, reworked by the German pandectistics, is currently being studied in the teaching plans of numerous Law Faculties in that country and has contributed to the current Chinese doctrine a universe of terms, concepts and legal categories that have been included in the new legislation, especially that relating to civil law.
}

Palabras claves: Derecho Romano, Derecho chino, República Popular China, recepción, Código Civil.

Keywords: Roman law, Chinese law, Chinese People's Republic, reception, Civil Code.

\section{Sumario:}

I. La influencia del Derecho Romano en la codificación del Derecho Civil chino.

II. La influencia del Derecho Romano en la ciencia jurídica china actual. 
III. La docencia del Derecho Romano en China.

Recibido: noviembre 2019.

Aceptado: enero 2020. 


\section{LA INFLUENCIA DEL DERECHO ROMANO EN LA CODIFICACIÓN DEL DERECHO CIVIL CHINO ${ }^{1}$}

A finales del siglo XIX China se abrió a la cultura jurídica occidental en su deseo de sentar las bases para la modernización del país siguiendo el modelo y el ejemplo de Japón ${ }^{2}$. Hasta entonces el Derecho chino, de tradición milenaria,

\footnotetext{
${ }^{1}$ Sobre la recepción del Derecho Romano en China, ya en la década de los años ochenta, podemos destacar las aportaciones de BEHRENDS, O., «Zur Lage des Römisches Rechts im heutigen China», en INDEX, Quaderni camerti di studi romanistici (Napoli), 14 (1986) 317 ss.; COLANGELO, L., «La ricezione del sistema giuridico romanistico e la relativa produzione di testi in Cina all'inizio del XX secolo: le fonti del diritto romano in due dei primi manuali in lingua cinese», en Bulletino dell'Istituto di Diritto Romano "Vittorio Scialoja” (B.I.D.R.) (Milano), 110 (2016) 196 nt. 2, quien nos ofrece una reseña muy reciente sobre los estudios en esta materia, dentro de los que destacamos al comienzo de este trabajo, las aportaciones del Profesor S., SCHIPANI, en la actualidad Profesor emérito en la Universidad de la Sapienza de Roma, y, también, profesor honorario de la Facultad de Ciencias Políticas y Derecho en la Universidad de Beijing (Pekín): SCHIPANI, S., s.v., "“Il diritto romano in Cina in XXI secolo. Norme e idee"», en Enciclopedia Treccani, Roma 2009, pp. 527 ss., ID., "Breve Nota", en Diritto cinese e sistema giuridico romanistico. Contributi, a cura di FORMICHELLA, L., TERRACINA, G., y TOTI, E., Torino 2005, pp. 9 ss. ; ID., "Il diritto romano in Cina", en AAVV., Diritto cinese e sistema ... cit., pp. 57 ss. [ = "Diritto romano in Cina", en Roma e America (Modena) 5 (1998)] ; ID., "Il diritto romano in Cina, Cina e diritto romano, ieri e oggi", INDEX (Napoli), 44 (2016) 501 ss. ; ID., "Fondamenti romanistici e diritto cinese (riflessioni su un comune lavoro nell'accrescimento del sistema)", en B.I.D.R. (Milano), 110 (2016) 7 ss.; ID., "Fondamenti romanistici e diritto cinese (un "tempo dei giuristi": riflessioni sull'accrescimento del sistema)", en Roma e America (Modena), 38 (2017) 93 ss. ; ID., "Fundamentos romanísticos y derecho chino (reflexiones sobre un esfuerzo común para ampliar el sistema)", en Revista de Derecho Privado (Bogotá), 35 (2018, julio/diciembre) 21 ss.: LIHONG ZHANG, Los últimos avances en la codificación del Derecho Civil chino en Revista Chilena de Derecho (Santiago de Chile), 3 (2009) 467 ss.

${ }^{2}$ Sobre el proceso de progresiva recepción del Derecho europeo occidental desde los primeros contactos en el siglo XVI hasta el siglo XX y, a través del mismo, de las bases del Derecho Privado Romano en Japón, PORCELLI, S., "Diritto cinese e tradizione romanistica. Terminologia e sistema", en B.I.D.R. (Milano), 110 (2016) 254 y 269 ss. Dicho Autor pone de relieve (o. c., p. 280), un dato muy significativo: en el año 1896, el gobierno japonés envió a Europa occidental a un grupo de 13 estudiantes a formarse en las universidades europeas, en 1906 ya fueron 1300, y en 1906 sumaban la importante cifra de 12000, de los cuales -PORCELLI-, dada la prioridad que tenían las reformas políticas y jurídicas en Japón, la mayor parte estudiaba ciencias políticas y jurídicas. Asimismo, en 1911 se habían traducido al japonés 958 libros
} 
se basaba en el $L i$, es decir, en los llamados ritos que integraban un derecho fundamentalmente consuetudinario, que regulaba las relaciones jurídicas de Derecho Privado sobre unas bases patriarcales y feudales y, por otra parte, el $\mathrm{Fa}$, es decir, la compilación de leyes escritas relativas al Derecho Penal y al Derecho Administrativo ${ }^{3,4}$. En este sentido, destaca FEI ANLING ${ }^{5}$ como caracteres que sintetizan la tradición jurídica china:

científicos procedentes de Occidente, y dentro de ellos 366 (el 38\%) eran obras relativas a las Ciencias Sociales. Véase también, COLANGELO, L., "Introduzione del diritto romano in Cina: evoluzione storica e recenti sviluppi relativi alla traduzione e produzione di testi e all'insegnamento", en Roma e America. Diritto romano comune (Modena), 36 (2015) 176-183.

Señala MI JIANG, "Diritto cinese e diritto romano", en Diritto cinese e sistema giuridico romanistico. Contributi, a cura di FORMICHELLA, L.; TERRACINA, G., y TOTI, E., Torino 2005, pp. 18 ss., que el 6 de abril de 1902 el emperador Guanxu de la dinastía Qing (16441911) publicó un edicto en el que ordenó el envío de juristas chinos de prestigio a diversos países de Europa y América para conocer el Derecho de Occidente. Entre ellos cabe destacar el viaje de Wu Tingfang a Inglaterra, Estados Unidos, España y Perú para estudiar el sistema político y jurídico de dichos países. A su retorno, dicho jurista fue nombrado en 1912 Ministro para la Revisión de las Leyes y, junto a Shen Jiaben, se encargó de la revisión de numerosas leyes de China, incluso llegó a ser Ministro de Justicia durante el gobierno provisional de Nankín. Hay que destacar con MI JIAN la participación directa de juristas japoneses en este proceso inicial de recepción del Derecho Romano en China, los cuales a su vez, estaban formados en el estudio del Derecho alemán, a partir del B.G.B. de 1900, dado que el sistema jurídico japonés moderno "fue fundado tomando directamente como modelo al sistema jurídico alemán" (o. c., p. 19), y así, no es de extrañar que el primer proyecto de Código Civil de China, elaborado a principios del siglo XX, al final de la dinastía Qing (1911), adoptase la estructura del Código Civil alemán, dividido en cinco partes. Sobre el estudio del Derecho Romano en las universidades de China a comienzos del siglo XX y la producción de manuales y estudios romanísticos en esta época, COLANGELO, L., La ricezione del sistema giuridico... cit., pp. 197 ss.; FORMICHELLA, L., "Fondamenti romanistici e insegnamento del diritto cinese", en B.I.D.R. (Milano), 110 (2016) 220 ss.

${ }^{3}$ En el sistema jurídico tradicional chino: a) los Ritos $(L i)$ eran los elementos principales de todas las normas de comportamiento social y estaban sustancialmente basados en las enseñanzas de Confucio. Junto a los castigos (Xing), ambos constituían la ley $(F a)$, con el significado moderno del término. Con los Ritos no sólo se indican ceremonias y costumbres fundadas sobre la cultura tradicional, sino también las normas de comportamiento basadas en los Tres Gang (que aluden a los lazos que unen los hilos de una red: el término fue acuñado por el filósofo confucionista Dong Zhongshu (179-104 a.C.) para indicar las tres relaciones sociales que están en la base de la ética social: entre soberano y súbdito, entre marido y mujer, y entre padre e hijo), los Cinco Chang (cinco virtudes constantes de la moral confucionista: humanidad, rectitud, observancia de los ritos, sabiduría y fidelidad) y en el sistema de relaciones de parentesco. Los castigos son complementarios a los Ritos de tal manera que "los Ritos son la cosa principal y los castigos son a ellos complementarios", "salir del ámbito de los Ritos es entrar en el campo de los castigos". b) Por otro lado, las leyes derivaban del emperador que encarnaba la voluntad celeste en su propia persona y era también el supremo administrador de la justicia. c) La ley no es igual para todos sino que siempre hubo en China una aristocracia que gozaba de privilegios incluso en el ámbito del Derecho Penal. d) Unión entre Administración y Jurisdicción existiendo una confusión en la persona del emperador entre los poderes legislativo, ejecutivo y judicial. MI JIAN, Diritto cinese... cit., pp. 15-16. YANG ZHENSHAN, "La tradizione filosofica del diritto romano e del diritto cinese antico e l'influenza del diritto romano sul diritto cinese 
contemporaneo, Diritto cinese", en Diritto cinese e sistema giuridico romanistico. Contributi, a cura di FORMICHELLA, L.; TERRACINA, G., y TOTI, E., Torino 2005, pp. 9 ss., p. 31.

${ }^{4}$ Sobre el derecho tradicional chino, véase los estudios llevados a cabo por MACCORMACK, G., : "The Law of Contract in China under the T'ang und Sung Dynasties", en Revue Internationale des Droits de l'Antiquité (R.I.D.A.) (Bruxelles), 32 (1985) 17 ss.; ID., Traditional Chinese Penal Law, Edinburgh, 1985; ID., "Hsiang Hsing and Hua Hsiang: The Problem of "Symbolic" Punishments in Early China", en R.I.D.A. (Bruxelles), 49 (2002) 297 ss.; ID., "The Transmission of Penal Law (lï) from the Han to the T'ang: A Contribution to the Study of the Early History of Codification in China", en R.I.D.A., (Bruxelles), 51 (2004) 47 ss.

Por ejemplo, MACCORMACK pone de relieve que en el derecho tradicional chino no se dio una verdadera regulación en sentido técnico de los contratos, los cuales no fueron objeto de una definición y sistematización en categorías por los juristas chinos. De esta forma, sólo podemos encontrar regulaciones normativas fragmentarias sobre cuestiones administrativas relacionadas con los contratos públicos realizados por funcionarios con los particulares, o normas relativas a los contratos en una localidad determinada, o referentes a tipos de contratos en particular, ya fuesen compraventas, préstamos o arrendamientos, dejando libertad a las partes para celebrar los acuerdos que estimasen convenientes. Y todo ello sin que llegase a existir un lenguaje técnico jurídico en esta materia. MACCORMACK, G., The Law of Contract... cit., pp. 18-19.

Por otro lado, MACCORMACK, G., The Law of Contract... cit. pp. 17-20, pone de relieve que en el derecho tradicional chino las normas emanadas de la autoridad imperial adoptaron la forma de estatutos $(L \ddot{u})$, ordenanzas (Ling) o regulaciones $\left(K^{\prime} O\right)$. Las primeras, dirigidas a regir materias de importancia para el Imperio, estaban destinadas a permanecer en vigor por mucho tiempo; en un grado inferior se encontraban las ordenanzas y regulaciones destinadas a cuestiones más puntuales o dictadas por exigencias del momento. Añade que, en general, dicho conjunto de reglas fueron recogidas en forma de códigos entendidos en sentido amplio, es decir, como meras recopilaciones de normas sobre una materia, realizadas en el curso de las sucesivas dinastías imperiales que rigieron la vida política de ese inmenso país a lo largo de su historia, pero, sin que las mismas siguiesen en su contenido normativo un verdadero orden sistemático por materias. Ahora bien, se dio el caso, verdaderamente aislado, de algún código que a lo largo de la historia de China reunió una cierta ordenación de las materias por capítulos y artículos, siendo el más importante el código de la dinastía T'ang, que se formó gradualmente entre los años 618 a 643 (d.C.) y que es conocido hoy en día por su edición del año 737. Aunque aparece formalmente como un código penal -y este fue su origen- lo cierto es que contenía, ordenadas en 12 capítulos integrados a su vez por artículos, materias de Derecho penal, procesal penal, administrativo, de familia, o de contratos, MACCORMACK, G., The transmission of Penal Law lü... cit., pp. 48-49; 73-78.

Destaca FEI ANLING, "Gli sviluppi storici del diritto cinese dal 1911 fino ad oggi", en Roma e America (Modena), 23 (2007) 112 ss, que el texto jurídico más antiguo en la historia de China es el Faijing (Clásico de la Ley), escrito por Li Kui (s. VI- V a.C.), una figura eminente de la escuela legalista. Esta escuela sostenía el establecimiento de una política de gobierno fundada sobre reglas jurídicas, en contraste abierto con la escuela confucioniana que defendía que el gobierno debía basarse sobre todo en reglas éticas y que el derecho tendría sólo un papel secundario y suplementario en la organización de la sociedad. El confucionismo fue durante largo tiempo la base de la ideología oficial china en esta materia pero la escuela legalista se reafirmó sobre todo en periodos de crisis o inestabilidad política y social en los cuales los gobiernos precisaban promulgar leyes punitivas para mantener el orden social. En este sentido -FEI ANLING, o. c., p. 112-, en la tradición china, cuando se habla de Derecho se piensa propiamente en Derecho penal.

${ }^{5}$ FEI ANLING, "Gli sviluppi storici del diritto cinese dal 1911 fino ad oggi”, en Roma e America (Modena), 23 (2007) 113. 
1. La elaboración jurídica realizada por los estudiosos constituyó una parte importante de los textos jurídicos, pero sus obras se concentraban, sobre todo, en la interpretación de las leyes.

2. No hubo una distinción neta entre los diversos sectores jurídicos, sin embargo, en el Ordenamiento Jurídico el Derecho Penal ha ocupado una posición dominante.

3. El Derecho Civil no ha tenido un desarrollo suficiente en China y, en este ámbito, los usos y costumbres han desempeñado un papel fundamental en la regulación de las relaciones humanas.

4. Los cambios de las diferentes dinastías gobernantes determinaron también profundos cambios en el Ordenamiento Jurídico: existió la práctica consolidada en virtud de la cual los nuevos gobernantes que se hacían con el poder en el país, acostumbraban a derogar las leyes anteriores y a crear un nuevo ordenamiento jurídico que legitimase su propia existencia.

La alternativa que se planteó a los juristas chinos de las primeras décadas del siglo XX para estructurar un sistema jurídico de Derecho Privado que sirviese de base para el nacimiento de una China moderna fue, o bien seguir el modelo del Common Law anglosajón, o bien el modelo representado por el Derecho europeo continental y, más concretamente, por el Código Civil alemán basado mayoritariamente en el Derecho Romano reelaborado y sistematizado por la pandectística alemana. Triunfó esta segunda opción ${ }^{6} \mathrm{y}$, conforme a la misma, comenzó a estudiarse el Derecho Romano en las Facultades de Derecho y, también, siguiendo este modelo de la Europa continental, de base romanista, fue elaborado el Código Civil de 1929-19317 , el cual fue derogado en 1949

${ }^{6}$ Como señala SCHIPANI, S., Fondamenti romanistici... cit., en Roma e America (Modena), 38 (2017) 111, a lo largo del periodo de la República (1911 -1949) llegó a haber en China unas 50 facultades de Derecho en las que se impartía la asignatura de Derecho Romano como asignatura obligatoria y se publicaron los primeros manuales de Derecho romano en lengua china a partir del año 1905. COLANGELO, L., Introduzione del diritto romano... cit., pp. 183 ss.

${ }^{7}$ Las sucesivas comisiones de juristas que visitaron Europa y América durante ese periodo dieron paso posteriormente a una importante actividad legislativa que estuvo muy influenciada por el Derecho japonés, ya que el Imperio del Sol Naciente había aprobado su Código Civil en 1869 que entró en vigor en 1898, tomando como base fundamentalmente las aportaciones de la pandectística alemana que -como sabemos y señalé en la nota 2-, partía del estudio de las fuentes jurídicas romanas para construir un sistema jurídico de Derecho Civil universal articulado en torno a una serie de conceptos y categorías jurídicas generales y abstractas. Sobre esta materia: DOMINGO, R., "El Código Civil japonés, un código a la europea", en Revista de la Facultad de Derecho de la UNED (Madrid), 21 (2003) 263 ss..

Tras la caída del último emperador y la instauración de la República china, fue aprobado en 1918 un segundo proyecto de Código Civil, también de base romanista, que estableció "las 
con el triunfo de Mao Zedong que impuso un régimen comunista, que tuvo una primera fase (1947-1959) de orientación soviética y, posteriormente, una segunda fase (1958-1977), más radical, en la que Mao llevó a cabo la llamada "revolución cultural"".

Tras la muerte de Mao Zedong y, sobre todo, con la llegada al poder de Den Xiao Ping, comenzó en 1978 una nueva era en la historia de China, de apertura hacia un régimen mixto entre la dirección política del Partido Comunista y la clara y decidida apuesta por un sistema económico de corte capitalista que conllevaba una evidente apertura hacia Occidente y una paulatina liberalización en los usos y costumbres sociales en una nación de más de mil millones de habitantes. En este ámbito, las élites dirigentes del país comprendieron que no puede haber un verdadero desarrollo económico ni un

\footnotetext{
bases del contenido y del sistema del derecho chino moderno". Dicho proyecto seguía la línea marcada por los juristas del último periodo de la dinastía Qing y, por tanto, estaba fuertemente influenciado por el Derecho japonés y alemán. Finalmente, tras décadas de trabajos preparatorios se aprobó el Código Civil de la nueva República china entre 1929 y 1931 (1930), siguiendo el modelo del Código japonés y alemán, pero influenciado también por el Código Civil de la Unión Soviética, e incluso, por el Código Civil tailandés. Dicho Código Civil chino de 1930 está actualmente básicamente en vigor, con algunas modificaciones, en la República de Taiwan. MI JIAN, Diritto cinese... cit., p. 21; HUANG MEILING, "Discussioni e tendenze nel processo di codificazione del diritto civile cinese", en INDEX (Napoli), 41 (2013) 586; SCHIPANI, S., Fondamenti romanistici... cit., en Roma e America (Modena), 38 (2017) 111; FEI ANLING, Gli sviluppi storici... cit., pp. 117 ss. Como señala también SCHIPANI, S., "Il diritto romano in Cina", en Diritto cinese e sistema giuridico romanistico... cit., p. 60, el primer libro de aquel código estaba dedicado a los principios generales y comprendía siete capítulos: sobre los principios, sobre las personas, sobre los bienes, sobre el negocio jurídico, sobre los plazos, sobre las prescripciones extintivas y sobre el ejercicio de los derechos. Los siguientes libros estaban dedicados a las obligaciones, a los derechos reales, a la familia y a las sucesiones. SCHIPANI subraya como el artículo 1 de dicho código sintetizaba el sistema de fuentes: ley, costumbre y ratio legis.

${ }^{8}$ Escribe JIAN PING, "Il diritto romano nella Repubblica Popolare Cinese", en Diritto cinese e sistema giuridico romanistico. Contributi, a cura di FORMICHELLA, L.; TERRACINA, G., y TOTI, E., Torino 2005, p. 1 ss. (p. 3), que "entre 1949 y 1957 la ciencia jurídica fue floreciente, pero tenía una visión completamente negativa tanto respecto del sistema y de la cultura jurídica de la antigua China como respecto del mundo occidental. Intentaron crear un nuevo sistema jurídico revolucionario según el ejemplo de la Unión Soviética y sobre la base de un completo rechazo de las concepciones de los países occidentales y en este marco también del Derecho Romano. Los cursos de Derecho Romano en las universidades fueron abolidos. En el segundo periodo de 1958 a 1977 se llegó a ignorar e incluso a negar completamente el papel del Derecho en general. Nosotros "los juristas chinos" lo llamamos el periodo del "nihilismo jurídico" habiéndose aprobado en el mismo poquísimas leyes y además, en la elaboración de las mismas, no se estudió el Derecho de otros países, ni siquiera el de la Unión Soviética. Durante la llamada revolución cultural el "nihilismo jurídico" alcanzó su punto álgido". SCHIPANI, S., Il diritto romano in Cina, Cina e diritto romano, ieri e oggi... cit., pp. 503-504; COLANGELO, L., Introduzione del diritto romano... cit., pp. 198 ss.; HUANG MEILING, Discussioni e tendenze nel processo... cit., pp. 587-588.
} 
auténtico progreso social sin la articulación legislativa de un sistema jurídico de Derecho Privado basado en principios de justicia y equidad y que reconozca la autonomía de la voluntad de los ciudadanos a la hora de realizar negocios jurídicos y para actuar con libertad en el tráfico jurídico y mercantil. Sin dicho marco legal no podía producirse el desarrollo económico y social deseado. De ahí que China volviese a mirar a Occidente, como ya sucedió casi un siglo antes, $\mathrm{y}$, precisamente, es en este contexto en el que ha surgido en las últimas décadas un notable interés hacia el Derecho Romano y su recepción en los ordenamientos jurídicos del continente europeo. En este sentido, recordamos con Schipani ${ }^{9}$ como, dentro de las resoluciones de política legislativa sancionadas en 1978, en el curso de la Tercera Sesión Plenaria del Comité Central elegido en el XI Congreso Nacional del Partido Comunista de China, se reconoció expresamente el "significado social general del Derecho Romano", "su racionalidad", "la posibilidad de ser heredado «recibido» y la utilidad que el mismo representa para la República Popular China dentro de su política de apertura a la problemática socialista de mercado".

En el plano legislativo se aprobó en 1986 la Ley de Principios Generales de Derecho Civil la cual establecía un catálogo de disposiciones en esta materia que pudiesen servir de fundamento para la posterior publicación de numerosas leyes de Derecho privado así como la elaboración de un Código Civil ${ }^{10}$, cuyo proceso

\footnotetext{
${ }^{9}$ SCHIPANI, S., Fondamenti romanistici... cit., en Roma e America (Modena), 38 (2017) 112; JIANG PING, "Il diritto romano nella Repubblica Popolare Cinese", en Diritto cinese e sistema giuridico romanistico. Contributi, a cura di FORMICHELLA, L.; TERRACINA, G., y TOTI, E., Torino 2005, pp. 3 ss. (= INDEX (Napoli), 16 (1998) 317 ss.).

${ }^{10}$ La aprobación de la Ley de Principios Generales de Derecho Civil de 1986 sirvió de base para la elaboración de una serie de leyes en este ámbito. La idea era que dichas leyes pudiesen ser luego reunificadas para la elaboración de un único texto como Código Civil de la República Popular China. Dicha ley se inspiró en el capítulo primero del Código Civil alemán y contiene 156 artículos englobados en nueve capítulos relativos, respectivamente, a las siguientes materias: "Principios generales" (arts. 1-8); "De la persona física" (arts. 9-35); "De la persona jurídica" (arts. 36-53); "Del negocio jurídico, de la representación" (arts. 54-70); "De los derechos civiles" (arts. 71-105); "Sobre la propiedad y otros derechos reales, obligaciones, propiedad intelectual, de la responsabilidad civil" (arts. 106-134); "De la prescripción" (arts. 135-141); "De la aplicación de la ley en las relaciones de Derecho civil con sujetos extranjeros" (arts. 142- 150); y "Disposiciones integradoras" (arts. 151-160). Véase, FEI ANLING, Gli sviluppi storici... cit., p. 122. Citamos también a YANG ZHENSHAN, La tradizione filosofica ... cit. p. 40, que realiza un estudio acerca de la influencia de los principios del Derecho Privado romano sobre los principios y normas contenidas en la Ley de Principios de 1986. El Autor parte de una reflexión fundamental: "la razón más profunda por la cual el derecho civil continental europeo hereda y desarrolla el derecho romano reside en el hecho de que las relaciones jurídicas entre productores de bienes que aparecen en el derecho romano son completamente conformes con las necesidades de la economía de mercado de la sociedad moderna". YANG ZHENSHAN sintetiza los tres principios elementales sobre los que se articula el Derecho Privado romano, frente a la tradicional sociedad feudal china que giraba en torno al poder del
} 
de elaboración ha sufrido diversas vicisitudes a lo largo de los últimos años estando prevista, a día de hoy, su aprobación definitiva a lo largo del año 2020; dicho Código Civil seguirá, en gran parte, en su estructura, la sistemática de los códigos civiles europeos y latinoamericanos ${ }^{11}$ de base romanista ${ }^{12}$. Señala XUE

emperador: la inviolabilidad de los derechos "de los ciudadanos", la igualdad de los sujetos ante la ley, y la autonomía de la voluntad, o. c., p. 36.

A partir de dicha Ley de Principios Generales y a la espera de la elaboración de un futuro Código Civil, se optó por llevar a cabo la aprobación de una serie de leyes sectoriales de Derecho Civil enmarcadas en el sistema normativo y conceptual de aquella ley: así podemos citar la ley sobre contratos de 1999, la ley sobre el matrimonio de 1980 modificada en 2001, la ley sobre sucesiones por causa de muerte de 2005, la ley sobre adopciones de 1991, modificada en 1998, la ley de procedimiento civil de 1991, modificada posteriormente en 2007 y 2012, la ley sobre derechos reales en 2007, la ley sobre la responsabilidad por ilícito civil de 2009 y la ley sobre la aplicación de las normas en las relaciones de Derecho civil con sujetos extranjeros, TIMOTEO, M., "La lunga marcia della codificazione civile nella Cina contemporanea", en B.I.D.R. (Milano), 110 (2016) 3.

${ }^{11}$ Si bien, TIMOTEO, M., La lunga marcia... cit., p. 44, pone de relieve en su estudio como en los últimos decenios numerosos juristas chinos también se han formado en las universidades de Estados Unidos, lo que se ha traducido en una recepción de principios, conceptos y categorías del Common Law en el contexto del derecho global contemporáneo y en el marco de la utilización mundial del inglés como lingua franca. Por ejemplo, en China aparecen dos conceptos, uno, de origen romanístico-civilístico, de responsabilidad sin culpa (no fault liability en terminología anglosajona), que los juristas chinos traducen por wu guocuo zeren, y otro, el concepto anglo americano de strict liability (yange zeren), si bien observa TIMOTEO, M., o. c., p. 44-, "en la ley china se utiliza la expresión wu guocuo zeren para referirse a la responsabilidad sin culpa, en la práctica en la literatura civilística china los dos conceptos se superponen a menudo sin atención a su significado jurídico, produciéndose numerosas confusiones a nivel no sólo de comunicación "interlingüística" entre juristas de nacionalidades diversas, sino también conceptuales dentro del ordenamiento chino". La misma Autora señala en un comentario reciente a la Parte General del Código Civil, que presumiblemente entrará en vigor en 2020, que junto a las bases pandectísticas presentes tanto en la estructura como en la dogmática terminológica y conceptual del nuevo Código Civil, también se aprecia una cierta influencia de principios y reglas procedentes del Common Law de Estados Unidos, como es, por ejemplo, en la recepción de los llamados daños punitivos para el caso de incumplimiento de obligaciones derivadas de la ley o de un contrato, dando lugar, por tanto, no sólo a la obligación de resarcir el daño producido por el incumplimiento y a la posible indemnización de perjuicios, sino también a la imposición de una verdadera pena pecuniaria a cargo del que incumple la obligación, TIMOTEO, M., o.c., p. 254. Y todo ello, sin olvidar el status especial de Hong Kong en el que sigue aplicándose un modelo basado en el Common Law británico, TIMOTEO, M., o.c., p. 258. En ese sentido, XU GUODONG, Note introduttive... cit., pp. 149- 150, destaca la labor realizada por la Columbia Law School y otras universidades americanas al becar a estudiantes chinos que se han formado en dichas universidades.

${ }^{12}$ XU GUODONG, "La base romanistica della parte generale del codice civile cinese", en B.I. $D$. R. (Milano), 110 (2016) 47 ss. , realiza un -a mi juicio- importante y clarividente comentario de diversos artículos del Proyecto de Código Civil chino, bien de su Parte General, bien de materias tales como obligaciones y contratos o derechos reales, para poner de relieve la base romanista que inspira dichos artículos. Por ejemplo, el artículo 16 de la Parte General establece que "por cuanto concierne a la tutela de los intereses del concebido que miran a la sucesión de la herencia, a la aceptación de un legado, etc..., se considera que el concebido tenga capacidad jurídica; 
JUN $^{13}$ que en 2014 por primera vez en la historia, el Partido Comunista Chino declaraba en un documento político titulado "Las decisiones del Comité Central del Partido Comunista Chino sobre los problemas fundamentales en la compleja construcción de un país cuyo gobierno se base en el respeto del Derecho $(F a)$ ", la necesidad de elaborar un Código Civil, "en cuanto que este constituye la base de todo el sistema jurídico chino". XU GUODONG ${ }^{14}$, profesor de Derecho de la Universidad de Xiamen, describe pormenorizadamente las vicisitudes y el iter de los trabajos preparatorios para la definitiva promulgación del Código Civil chino concluyendo dicho camino el día 15 de marzo de 2017 en el que la V Sesión Plenaria de la XII Asamblea Nacional Popular votó a favor de la promulgación de la Parte General del Código Civil Chino (Minfa Zongze), de 206 artículos integrados en 11 capítulos, la cual constituye el libro primero del Código Civil, cuya entrada en vigor está prevista el año $2020^{15}$. El Proyecto relativo

pero si el concebido no está vivo en el momento del nacimiento se entiende que no tuvo capacidad jurídica desde el inicio". Añade el Autor que la base de este artículo se encuentra en D. 1,5,26 (Iul., 69 dig.); XU GUODONG, La base romanistica ... cit., pp. 58-61. También, JIANG PING, "Lo sviluppo della elaborazione del codice civile cinese e le principali controversie in dottrina in merito (breve nota informativa)", en Roma e America (Modena), 17 (2004) 347-351.

Una vez publicada la Parte General del Código Civil chino, han continuado los trabajos para la elaboración de los cinco libros que integrarán el contenido normativo, por materias, de dicho código: derechos reales, contratos, responsabilidad civil, familia y sucesiones. A este respecto XUE JUN, "La codificazione del diritto civile cinese e il diritto romano", en B.I.D.R. (Milano), 110 (2016) 73 ss. 79, realizó una interesante comparación entre la labor de los codificadores chinos en la actualidad con los compiladores de Justiniano señalando que "este programa de trabajo «de los codificadores chinos» se basa aún en la idea del código como compilación, en cuanto que cada libro de la parte especial se corresponde con leyes sectoriales emanadas en los últimos años: el libro relativo a los derechos reales se corresponde con la ley sobre derechos reales del 2007, el libro sobre los contratos se basa en la ley de contratos de 1999, el libro de responsabilidad civil deriva de la ley de responsabilidad civil de 2009, el libro sobre la familia hace referencia a la ley de matrimonio de 1980, el libro sobre sucesiones se funda sobre la ley de sucesiones de 1985".

${ }^{13}$ XUE JUN, La codificazione... cit., p. 76.

${ }^{14}$ XU GUODONG, La base romanistica... cit., pp. 48-49; ID., "Note introduttive all'esame della struttura dei tre principali progetti di codice per la RPC attualmente in fase di elaborazione", en Roma e America (Modena), 23 (2007) 133 ss., llevó a cabo un amplio y detallado estudio sobre la marcha de los trabajos encaminados a la redacción del nuevo Código Civil chino y de las divergencias que han ido surgiendo en muchos casos, debido a las distintas influencias y concepciones políticas y jurídicas que se dan entre los juristas chinos contemporáneos.

15 TIMOTEO, M., "La parte generale del codice civile cinese fra modelli importanti e modeli locali", en Roma e America (Modena) 245 -247, pone de relieve que los once capítulos que integran la Parte General siguen el modelo de la pandectística alemana: Disposiciones generales (arts. 1-12); Personas físicas (arts. 13- 56); Personas jurídicas (arts. 57-101); Organizaciones sin personalidad jurídica (arts. 101-108); Derecho civiles (arts. 109-132); Negocio jurídico civil (arts. 133-160); Representación (arts. 161-175); Responsabilidad civil (arts. 176-187); Plazos para actuar en juicio (arts. 188-199); Modalidad de cómputo de los plazos (arts. 200204); Disposiciones supletorias (arts. 205-206). 
a los libros sucesivos, titulado Partes especiales del Código Civil se presentó a la Comisión Legislativa de la Asamblea Nacional del Pueblo el 27 de agosto de 2018 y fue publicado el 17 de septiembre del mismo año ${ }^{16}$.

\section{LA INFLUENCIA DEL DERECHO ROMANO EN LA CIENCIA JURÍDICA CHINA ACTUAL}

El sistema de fuentes del Derecho Romano es en la actualidad motivo de estudio y de comparación con el sistema de fuentes actualmente vigente en China. Así, XUE JUN ${ }^{17}$ después de poner de relieve que las sentencias de la Corte Suprema de China tienen un valor meramente interpretativo e integrador del Ordenamiento Jurídico, señala que "en la doctrina se da una polémica sobre la naturaleza jurídica de las reglas surgidas de la interpretación judicial que de las leyes realiza dicho órgano judicial: una parte de los estudiosos entiende que, como la Corte Suprema es un órgano judicial y no legislativo, no se debe reconocer vigencia general y plena a las reglas creadas por la Corte Suprema"; y añade que "las reglas surgidas de las interpretaciones judiciales pueden ser definidas como costumbres judiciales que no tienen valor para derogar de facto las normas establecidas en la ley".

Y a continuación, indica el Autor que, sobre este punto, puede servir de reflexión el dualismo existente en el Derecho Romano entre el ius civile y el ius honorarium, desde el momento en que el fenómeno de las "interpretaciones judiciales" en China tiene funciones y caracteres muy similares a los del ius

A este respecto, SCHIPANI, S., Fondamenti romanistici... cit., Roma e America (Modena), 38 (2017) 118 , constata que a lo largo de estos últimos años se ha producido un cambio sustancial en la percepción de principios generales del Derecho en torno a los cuales debe articularse el Derecho Privado de la RPC. Así, el artículo 2 de la Ley de Principios Generales del Derecho Civil de 1986 ponía como punto central digno de protección jurídica al patrimonio al decir que "el derecho civil regula las relaciones patrimoniales y las personales", mientras que el artículo 2 del Código Civil aprobado señala que "el derecho civil regula las relaciones personales y patrimoniales entre personas físicas y/o jurídicas y/o organizaciones sin personalidad jurídica como sujetos en posición de paridad". Señala SCHIPANI que el texto del nuevo Código, que sobre este punto sigue las aportaciones de los sucesivos proyectos del Código Civil, al poner las relaciones personales en primer lugar, "accoglie l'indicazione di Inst. Iust., 1,2,12, come anche di Digesto 1,5,2, che pongono i rapporti personali, come primo oggetto del diritto civile, e a seguire pongono $i$ rapporti patrimoniali, per $i$ fatto che $i$ rapporti che riguardano le proprietà sono funzionali a quelli che riguardano le persone, il che rappresenta una prospettiva umanistica del diritto civile (vid. también, XU GUODONG, La base romanistica... cit., pp. 49 ss., p. 54.

${ }^{16}$ TIMOTEO, M., La parte generale... cit., 247.

${ }^{17}$ XUE JUN, La codificazione... cit., p. 82; en este sentido, véase también, YIN QIUSHI, "Le interpretazioni della Suprema Corte del popolo cinese e lo ius honorarium", en Roma e America (Modena), 37 (2016) 251 ss. 
honorarium en la experiencia romana. Así, pone de relieve que en el sistema constitucional romano, los magistrados tenían funciones jurisdiccionales, y en particular los pretores urbani y peregrini, ejercitaban una función "nomogenética" muy relevante creando el gran bloque de normas denominado ius honorarium que se desarrollaba en una posición paralela al ius civile. En China las "interpretaciones judiciales" de la Corte Suprema llevan a cabo una función muy relevante en el desarrollo, integración y mejora de cuanto esté dispuesto en las leyes aprobadas por los órganos titulares de la función legislativa. De esta forma, "si bien, formalmente, en el ordenamiento constitucional, la Corte Suprema no es un órgano legislativo, "ella, spinta dalle necessità della realtà", realiza la función de desarrollar las normas de modo muy activo dando vida, de esta manera, a la versión china del ius honorarium". Y no sólo esto, sino que subraya Xue Jun ${ }^{18}$ - la experiencia romana del ius honorarium y su historia de "codificación" han inspirado a los juristas a madurar la idea de una codificación en la cual pueden confluir las leyes y las interpretaciones judiciales. Así, en el Derecho Romano, el ius honorarium permaneció en un primer término como una parte del derecho separada del sistema del ius civile, pero el trabajo de la ciencia jurídica ya en época clásica estaba destinado a fundir los dos complejos de normas que culminan en la Compilación de Justiniano.

Por otro lado, JIANG PING ${ }^{19}$ destaca diferentes aspectos del Derecho Privado Romano, como son el principio de buena $\mathrm{fe}^{20}$, la capacidad jurídica tanto de

\footnotetext{
${ }^{18}$ XUE JUN, La codificazione... cit. p. 83.

19 JIANG PING, "Il risorgere dello spirito del diritto romano in Cina", en Diritto cinese e sistema romanistico. Contributi, a cura di FORMICHELLA, M., TERRACINA, G., TOTI, E., y Torino 2005, pp. 53-54, indica que "el principal mérito del ius naturale romano es el haber exaltado la individualidad y haberla liberado del sometimiento a la autoridad social. En el Derecho Romano, frente a la originaria supremacía de la familia, entró la del individuo, lo que llevó, a su vez, al nacimiento del Derecho Privado y a la consecuente igualdad jurídica. La sociedad romana, en cuanto esclavista, habría debido dar vida a un derecho fundado en la desigualdad, pero, por el contrario, dio lugar al Derecho Privado, que es la mejor manifestación del espíritu de igualdad. En realidad, la sociedad romana reflejó en el derecho su doble naturaleza: de un lado, el derecho contractual que expresa relaciones comerciales paritarias entre los hombres libres, y por otro, el derecho familiar, afirmando la centralidad del pater familias y de su potestas, encarnando la desigualdad entre personas. El ius gentium, que reflejaba el espíritu del derecho natural, no estaba sujeto a vínculos del sistema familiar dominado por la patria potestas. En esta óptica se puede decir que en el Derecho Romano tuvo lugar un fenómeno de paso del status al contrato".

${ }^{20}$ El art. 5 de la Ley de Principios Generales del Derecho de 1986 establece que "los derechos civiles y los intereses legítimos de los ciudadanos y de las personas jurídicas son tutelados por las leyes. Ninguna organización o persona privada puede atentar contra ellos", JIANG PING, Il diritto romano nella Repubblica ... cit. p. 6, ve el origen de estas concepciones en formulaciones provenientes del Derecho Romano que no se daban en el Derecho chino tradicional, las cuales darían lugar, por ejemplo, a las leyes que dentro del Ordenamiento Jurídico de China luchan contra la competencia desleal entre empresarios.
} 
las personas físicas como de las jurídicas ${ }^{21}$, el régimen jurídico configurado para los derechos de propiedad, de usufructo, de superficie o de enfiteusis, que resulta de interés en China ya que ofrece construcciones jurídicas y soluciones útiles en el marco de la propiedad inmobiliaria estatal ${ }^{22}$, un análisis de los diferentes tipos de contratos consensuales y reales aplicables al tráfico mercanti1 ${ }^{23}$, o el estudio de los supuestos de responsabilidad civil derivados de delitos o de quasi-delitos ${ }^{24}$. Y todo ello en el marco de las concepciones filosóficas y humanistas propias de los juristas clásicos romanos.

${ }^{21}$ En la base está la configuración del individuo dotado de capacidad, reconocida por el Ordenamiento Jurídico, para actuar con libertad en los negocios jurídicos. Dichas construcciones referidas a las personas jurídicas han resultado de mucho interés para la regulación jurídica de las distintas formas de empresas reconocidas en China: hacienda agraria, hacienda familiar, hacienda individual, empresa privada, empresa estatal, empresa colectiva, empresa cooperativa, empresa asociativa, etc..., de las cuales -JIANG PING, Il diritto romano nella Repubblica... cit., p. 7-, no todas ellas gozan de personalidad jurídica y no todas de entre ellas ostentan una capacidad jurídica plena, mientras que, por el contrario, todas las personas físicas gozan de capacidad jurídica plena. A este respecto, expone FEI ANLING, "La persona giuridica nei progetti di codice civile della Cina: discussioni e prospettive", en B.I.D.R. (Milano), 110 (2016) 87-102, los trabajos de la comisión encargada de la redacción de los artículos del Código Civil relativo a las personas jurídicas.

${ }^{22}$ JIANG PING, o. c., p. 9. También, MI JIAN, “L'ususfructus e la possibilità di risolvere un problema dei Diritti reali in Cina. Spunti raccolti dal diritto romano e dal codice civile italiano, en Roma e America (Modena), 5 (1998) 267 ss.; VINCI, M., "La servitù prediali in Cina e la funzione integratrice del Diritto romano (prime riflessioni), en Roma e America (Modena), 28 (2009) 183 ss.

${ }^{23}$ En este sentido, la ley de Principios de 1986 seguía la estructura del BGB de 1900 y así reservaba un capítulo para el "acto jurídico", en el que se comprende el contrato como acuerdo de voluntades entre las partes y se regulan las condiciones para que un contrato pueda ser considerado válido o inválido, así como la eficacia de los mismos. En otro capítulo se incluyen las obligaciones derivadas de los contratos, su ejecución y garantías, y un tercer capítulo dedicado a la responsabilidad civil que recoge lo relativo al incumplimiento total o parcial de las obligaciones y a la responsabilidad por incumplimiento. JIANG PING, Il diritto romano nella Repubblica... cit., pp. 9-10; Veáse FORMICHELLA, L., Fondamenti romanistici... cit., p. 238, señala a este respecto que se aprecia en los juristas que redactaron la Ley de Principios de 1986, un sistema de responsabilidad contractual reconducible en ciertos términos a las reglas romanas sobre responsabilidad por dolo y culpa contenidas en D. 50,17,23. Sobre esta materia, también: JIANG PING, "Proyecto de ley contractual unitaria de la República Popular China", en Roma e America (Modena) 5 (1998) 243 ss.; RESCIGNO, P., "Appunti sulla legge della Repubblica Popolare cinese sui contratti”, en Roma e America (Modena), 8 (1999) 219 ss.; SCHIPANI, S., "La nuova legge cinese in materia di contratti e il diritto romano come base di essa e della comunicazione con i codici del sistema romanistico", en Roma e America (Modena), 8 (1999) 225 ss.

${ }^{24}$ La ley de Principios Generales recoge la distinción entre ilícito extracontractual derivado del concepto romano de delictum, de lo que es el incumplimiento en el ámbito de los contratos. Así, en la Ley de Principios, la responsabilidad civil por ilícitos penales y quasi delitos se recoge en el capítulo relativo a la responsabilidad civil y no la que se ocupa de las obligaciones, JIANG PING, Il diritto romano... cit., p. 10; JUN LI, La obbligo di diligenza nella responsabilità... cit., pp. 247 ss., p. 252. 


\section{LA DOCENCIA DEL DERECHO ROMANO EN CHINA}

Pone de relieve Formichella ${ }^{25}$ que ya en 1978 había cinco institutos universitarios en Pekín, Xi'An, Chongqing, Wuhan y Shanghai en los cuales se impartía docencia de Derecho Romano y que hoy en día podemos constatar la existencia de más de 600 Facultades o Institutos de Derecho en China, en muchos de los cuales se imparte docencia de Derecho Romano, en algunos como asignatura optativa autónoma y en otros formando parte del Curso de Historia de los Sistemas Jurídicos Extranjeros ${ }^{26}$.

Por lo que hace a la publicación de bibliografía romanística en China, Colangelo $^{27}$ señala que el primer manual de Derecho Romano escrito en este país fue el titulado: Luoma Fa (Derecho Romano), de Haung Youchang (1915), el cual fue objeto de numerosas reediciones. Asimismo, desde los años ochenta del pasado siglo se han publicado numerosas obras, entre artículos, manuales, monografías y traducciones de fuentes latinas (incluyendo la Compilación de Justiniano) y de autores extranjeros sobre la materia ${ }^{28}$, entre los que destacamos la traducción en lengua china de los dos primeros volúmenes de la Storia della

${ }^{25}$ FORMICHELLA, L., Fondamenti romanistici... cit., p. 222.

${ }^{26}$ FORMICHELLA, L., Fondamenti romanistici... cit., p. 226.

${ }^{27}$ COLANGELO, L., Introduzione del diritto romano... cit., pp. 196-197.

${ }^{28}$ Señala FORMICHELLA, L., Fondamenti romanistici... cit., p. 222, que "el primer escrito del cual tenemos noticia es una recopilación de materiales realizada bajo el título Luoma fa (Derecho Romano) realizada por Zhou Nan, Wu Wenhan y Xie Bangyu (1983), en la cual los autores realizan una amplia reconstrucción histórica del Derecho Romano siguiendo la tripartición de personae, res y actiones. Zhou Nan es el autor del manual históricamente más célebre, realizado inicialmente bajo la forma de apuntes que resumen 50 años de estudio y enseñanza por parte de uno de los precursores del Derecho Romano en China. Siguen Luoma fa Yuanli (Manual de Derecho Romano) (1988), de Qu Keshen; Luoma fa Tiyao (Elementos fundamentales de Derecho Romano) (1988), obra de Zhou Nan; Faxue zong lu-faxue jieti (Instituciones de Justiniano) (1989), traducidas por Zhang Qitai; Luoma fa (Derecho Romano) (1990), de Xie Bangyu; las traducciones realizadas por Huang Feng de las Istituzioni di diritto romano de Bonfante (Luoma fa Jiaokeshu) (1992), de la Storia del diritto romano de Grosso (Luoma fa shi) (1994), y de las Instituciones de Gayo (1997); la traducción de las Instituciones de Justiniano (Faxue Jieti /[Gu Luoma] Youshidingni zhe), por obra de Xu Guodong (1999); Luoma Sifa Daolun (Manual de Derecho Privado Romano) realizado por Haung Feng (2003); una ulterior edición de Luoma fa (Derecho Romano) de Qiu Hanping (2004); Luoma fa yuanli (Instituciones de Derecho Romano) (2006), de Chen Chaobi; Luoma fa yu Xiandai (Derecho Romano y moderno), de Huang Youcheng (2008); Luoma sifaxue (Manual de Derecho Privado Romano) (2009), de Fei Anling; la traducción de la Storia della costituzione romana de De Martino, vol. 1 y 2 de Xue Jun (2009 y 2015); Luoma Fa (Derecho Romano), de Meng Zhenxiang (2009); el más reciente texto de Xu Guodong, Luoma gongfa yaolun (Elementos de Derecho Público Romano) (2014)". También véase, TERRACINA, G., "Bibliografia romanistica pubblicata in China (1978-2003)", en INDEX (Napoli), 32 (2004) 259 ss..; ID., "Introduzione alla Bibliografia romanistica pubblicata in Cina (1978-2003)", en Roma e America (Modena), 17 (2004) 371-381. Véase también, COLANGELO, L., Introduzione del diritto romano... cit., p. 201 ss., 207. 
costituzione romana de Francesco de Martino, realizada por el Prof. Xue Yun de la Universidad de Pekín, siendo el primer volumen de esta obra incluido por el Prof. Liu Suli entre los cien mejores libros publicados en China en el año 2009 escogidos cuidadosamente entre los aproximadamente 110.000 libros publicados en dicho país a lo largo de ese año ${ }^{29}$.

Asimismo, en las últimas décadas, numerosos estudiantes y profesores de Derecho Romano de la República Popular China han realizado estancias docentes e investigadoras en universidades europeas, especialmente en las universidades de La Sapienza y Tor Vergata de Roma. En este sentido, se constituyó en 2008 entre las Universidades "La Sapienza" y "Tor Vergata" de Roma, y la Universidad china de Ciencias Políticas y Derecho de Pekín el "Osservatorio sulla codificazione e formazione del giurista in Cina nel quadro del sistema romanistico", cuyas actividades se dirigen, principalmente en tres direcciones: investigación científica, formación en grado y posgrado, y documentación. También en enero del 2017, se firmó en presencia del Prof. Sergio Mattarella, Presidente de la República Italiana, un convenio entre la Universidad de La Sapienza de Roma y la Zhongnan University of Economics and Law de Wuhan, por el que se creó el Centro di Studi Giuridici Italo Cinese, ubicado en el Aula Pietro Bonfante de Dirittto Romano de la Facultad de Derecho dicha universidad ${ }^{30}$.

${ }^{29}$ XUE JUN, "La traduzione cinese di "Storia della costituzione romana": il valore e l'attualità", en Roma e America (Modena ), 30 (2010) 235 ss. (235). A raíz de dicha publicación aparecieron distintos comentarios a la misma: CASAVOLA, F.P., "In occasione della traduzione in lingua cinese della storia della costituzione romana di Francesco de Martino", en Roma e America (Modena), 30 (2010) 211- 215; LABRUNA, L., "De Martino, la "Storia", la Cina", en Roma e America (Modena), 30 (2010) 217-222; VALDITARA, G., "Sulla traduzione al cinese della Storia della Costituzione Romana di De Martino", en Roma e America (Modena), 30 ( 2010) 223-231.

${ }^{30}$ DILIBERTO, O., "Chiausura dei lavori. Diritto romano e codificazione cinese tra passato presente e futuro. Alcuni considerazioni”, en B.I.D.R. (Milano), 110 (2016) 293 ss. 
